\title{
Seasonal variation in heavy metal accumulation in plants at coal mine sites and possible health risk
}

\author{
Ogbonna P. C. ${ }^{1, *}$, Nzegbule E. C. ${ }^{1}$ and Okorie P. E. ${ }^{1}$ \\ ${ }^{1}$ Department of Environmental Management and Toxicology, Michael Okpara University of Agriculture, \\ Umudike, Umuahia, Abia State, Nigeria \\ Corresponding Author: *ogbonna_princewill@yahoo.com
}

\begin{abstract}
Human health challenges in recent times have been attributed to consumption of food contaminated with heavy metals. Coal mining is an anthropogenic activity that releases heavy metals into the environment. This study, therefore, assessed accumulation of heavy metals (lead (Pb), cadmium $(\mathrm{Cd})$, nickel (Ni), arsenic (As) and iron (Fe)) in plant species in the vicinity of Akwuke and Iva coal mine in Coal city, Enugu State. The results indicate that the highest concentration of Ni $19.02 \pm$ $0.03 \mathrm{mg} / \mathrm{kg})$, As $(0.005 \pm 0.001 \mathrm{mg} / \mathrm{kg})$ and $C d(2.16 \pm 0.07 \mathrm{mg} / \mathrm{kg})$ was accumulated in Alchornea cordifolia while the concentration of $P b(10.37 \pm 0.52 \mathrm{mg} / \mathrm{kg})$ was highest in Andropogon gayanus and $\mathrm{Fe}(231.00 \pm 1.41 \mathrm{mg} / \mathrm{kg})$ was highest in Irvingia gabonensis. The values of $\mathrm{Ni}(9.02 \pm 0.03$ $\mathrm{mg} / \mathrm{kg})$, As $(0.005 \pm 0.00 \mathrm{mg} / \mathrm{kg}), \mathrm{Pb}(10.37 \pm 0.32 \mathrm{mg} / \mathrm{kg}), \mathrm{Cd}(2.02 \pm 0.03 \mathrm{mg} / \mathrm{kg})$, and $\mathrm{Fe}(46.00 \pm$ $1.41 \mathrm{mg} / \mathrm{kg}$ ) accumulated in A. cordifolia, A. gayanus, D. guinense, and M. indica in wet season were significantly $(P<0.05)$ higher than their corresponding values $(5.04 \pm 0.07,8.96 \pm 0.01$ and $0.003 \pm 0.001 \mathrm{mg} / \mathrm{kg}, 1.87 \pm 0.07 \mathrm{mg} / \mathrm{kg}$, and $33.45 \pm 0.21 \mathrm{mg} / \mathrm{kg}$ ) in dry season, respectively. Alchornea cordifolia, Andropogon gayanus and Irvingia gabonensis consumed by animals and local inhabitants in the vicinity of the two mined sites could expose them to high levels of $\mathrm{Pb}, \mathrm{Ni}$ and $C d$, thus posing a serious health risk to the local people.
\end{abstract}

Keywords: Seasonal variation, heavy metal, plants, coal mine, Enugu, Nigeria

\subsection{Introduction}

Human health challenges in recent times have been attributed to consumption of food contaminated with heavy metals. Food contamination is becoming very alarming due to high rate of exploitation of mineral resources such as coal, petroleum, stones (i.e. quarrying) among others. Coal mining is the process of extracting coal minerals from under the ground (Ogbonna et al., 2012) and this anthropogenic activity constitutes environmental problem since mining removes part or all of the standing plant material and litter, as well as the soil organic matter (SOM) in the surface horizons. Mining operations generates tailings that are deposited around mines and part of the tailing dust escape into the atmosphere. Thus, the atmosphere is a pathway for transport of heavy metals and the major input of bio-available metals in the environment, which are potential threats to the health and survival of man (Ogbonna et al., 2018b). For instance, the inhalation of cadmium (Cd) causes bronchitis, pneumonitis and inflammation of the liver (Mac Farland, 1979) but the effect becomes fatal and deleterious when up to $8 \mathrm{mg} / \mathrm{m}^{3}$ of cadmium oxide fume is inhaled for about 5 hours (Ademoroti, 1996). Cadmium is an enzyme inhibitor, having affinity for other ligands in plants and animal cells like carboxyl, crysteinyl and histidyl side chains of protein, porphyrin and purines. The symptoms of $\mathrm{Cd}$ toxicity produced by enzymatic inhibition include respiratory disorders, aminoaciduria (urinary excretion of excessive calcium), formation of kidney stones and damage to kidney and liver (Manzur, 1981). Argon (Ar) exert deleterious effect on the skin and this include hyperkeratosis on soles of feet and palms, hyper pigmentation in areas not exposed to the sun and skin cancer after prolonged exposure (Berman, 1980), causes cirrhosis (liver disease) and a rare form of liver cancer called haemogioendothelioma (Hutton, 1986). 
Heavy metals are either deposited directly on plant surfaces such as leaves, flowers, branches, and stems or on soils, which are absorbed from the soil solution into plants via the roots (Ogbonna et al., 2018b). Since herbivores largely depends on fodder plants for food and man also relied heavily on plants for nutrition and health benefits, heavy metal contamination of plants will constitute serious health risk to both man and animals residing around coal mine sites. A literature search showed that no work has been carried out on heavy metal pollution in seven plant species growing on or near coal mine-contaminated sites in Nigeria. The seven species sampled in this study (Irvingia gabonensis, Dialium guinense, Mangifera indica, Icacina trichantha, Alchornea cordifolia, Andropogon gayanus, and Manihot esculenta) are very important source of nutritional fruits (Mangifera indica, Irvingia gabonensis, Dialium guinense), medicine (Icacina trichantha), condiment (Irvingia gabonensis) and staple food (Manihot esculenta) for man while Andropogon gayanus and Alchornea cordifolia are important fodder plants for herbivores in South east Nigeria. The objective of this study is to investigate the level of concentrations of heavy metals in plants around the coal mine sites and compare the values with the maximum permissible limits of FAO/WHO. The results of this research will provide the background information on the levels of concentrations of heavy metals in the plants and serve as an important document for Enugu State Agricultural Development Programme to carry out extension services to the rural farmers on the possible danger associated with using the lands around the coal mine sites for agricultural purposes or utilizing the plants that are naturally growing there for nutritional and health purposes.

\subsection{Methodology}

\subsection{Study area}

The study on the determination of heavy metal accumulation in plants at coal mine sites was carried out at Enugu, Enugu State. The study covered both Akwuke and Iva mines. Enugu is the capital of Enugu State, south east Nigeria and has a population of 722, 000 in 2006 (NPC, 2006) and a large deposit of sub-bituminous coal. It lies within latitude $6^{\circ} 23^{\prime}$ and $6^{\circ} 26^{\prime} \mathrm{N}$ and longitude $7^{\circ} 27^{\prime}$ and $7^{\circ}$ $30^{\prime} \mathrm{E}$ (Ogbonna et al., 2018a) and the mean monthly temperature lies between 27 and $29^{\circ} \mathrm{C}$ (Ekere and Ukoha, 2013). The area has tropical climate and experiences two seasons both of which are warm. The wet season begins from April and ends in October while the dry season begins from November and ends in March (Ogbonna et al., 2012). The natural vegetation is tropical rain forest type but has significantly changed over time to guinea savannah due to human activities such as farming (Ezeigbo and Ezeanyim, 1993), nomadism and exploitation of fuel wood (Ogbonna et al., 2018a), erosion menace and rapid increase in human population. The three largely conformable geologic formations are Enugu shale (Campanian), the Mamu Formation (Lower Maastrichtian) and the Ajali sandstone (Upper Maastrichtian) which constitutes the geology of the Enugu coal mine area. The food and cash crops produced by farmers include yam, maize, ogbono (Irvingia gabonensis var excelsa), melon, cassava, local beans, oil palm, rice, groundnut, and cocoa (Ogbonna et al., 2018a).

\subsection{Sample collections}

Prior to the sample collection, a reconnaissance survey was carried out to determine the altitude of the mined sites (251 and $259 \mathrm{~m}$ for Akwuke and Iva mines, respectively) and plant species that were common at the two mined sites since mined sites are very slow in vegetation regrowth. The control samples were taken from a 5 year upland bush fallow that is $2 \mathrm{~km}$ away from the abandoned mines.

\subsubsection{Plant sampling and analysis of heavy metals}

In this study, plant sampling for determination of heavy metals content in plants was carried out on individual plant species that had at least 3 frequency of occurrence in each of the mined (Akwuke and Iva) and unmined (control) sites. Fresh leaves were sampled from different shoots/branches and parts of different woody species 7-9 years of age, except for root crops (cassava) and grass (gamba grass) that is less than 2 years. The leaves of Andropogon gayanus (gamba grass, family- Poaceae), Irvingia gabonensis (wild bush mango, family- Irvingiaceae), Dialium guinense (wild black velvet, velvet tamarind, family- Fabaceae), Mangifera indica L. (mango, family- Anacardiaceae), Alchornea cordifolia (Schum. \& Thonn.) Muell. Arg. (Christmas bush, family- Euphorbiaceae), Icacina trichantha Oliv. (pflamzenfen, family- Icacinaceae), and Manihot esculenta (cassava, familyEuphorbiaceae) were randomly collected in February (dry season) and June (wet season) separately 
from each individual plant using well-cleaned secateurs at the two mined locations and an unmined plot (a 5 year upland bush fallow that is $2 \mathrm{~km}$ from the mined sites where there was no visible source of contamination). Three replicates of each plant species were collected and mixed separately to obtain a composite sample. The plant samples were cleaned sequentially with a phosphate-free detergent (Extran 2\%), rinsed once with tap water, once with distilled water and finally twice with deionized water to remove adhering materials such as dust and pollen particles and placed in large clean crucibles where they were oven dried at $60^{\circ} \mathrm{C}$ for $72 \mathrm{~h}$. Thereafter, the dried plant samples were milled with a Thomas Wiley milling machine (Model ED-5 USA) to fine powder. The procedure according to Awofolu (2005) was used for digestion of plant sample. A $0.5 \mathrm{~g}$ of each sieved leaf samples was weighed into $100 \mathrm{ml}$ beaker. A mixture of $5 \mathrm{ml}$ concentrated trioxonitrate (IV) acid and $2 \mathrm{ml}$ perchloric acid was added and digested at $80^{\circ} \mathrm{C}$ using hot plate until the content was about $2 \mathrm{ml}$. The sample was allowed to cool, filtered into $50 \mathrm{ml}$ standard flask using 0.45 um Millipore filter kit. The beaker was rinsed with small portions of doubled distilled water and then filtered into the flask.

\subsection{Heavy metal content}

The concentrations of lead $(\mathrm{Pb})$, cadmium $(\mathrm{Cd})$, arsenic $(\mathrm{As})$, iron $(\mathrm{Fe})$, and nickel $(\mathrm{Ni})$ in the digested samples were determined using flame Atomic Absorption Spectrophotometer (AAS) of UNICAM 919 model. Triplicate digestion of each sample was carried out and blanks were prepared from only reagents without sample to check for background contamination by the reagents. Appropriate quality assurance procedures and precautions were taken to ensure the reliability of the results. Samples were carefully handled to avoid cross-contamination. Glassware was properly cleaned, and reagents used were of analytical grades. Deionized water was used throughout the study. Working standard solutions of $\mathrm{Pb}, \mathrm{Cd}, \mathrm{As}, \mathrm{Fe}$, and $\mathrm{Ni}$ were prepared from the stock standard solutions containing 1000 ppm of element in $2 \mathrm{~N}$ nitric acid. Calibration and measurement of elements were done on flame Atomic Absorption Spectrophotometer (UNICAM 919 model). A blank reading was also taken and necessary correction was made during the calculation of concentration of various elements.

\subsection{Experimental design and statistical analysis}

A single factor experiment was conducted in a randomized complete block design (RCBD) with three replications. Data collected was subjected to analysis of variance (ANOVA) using Statistical Package for Social Sciences (SPSS) v. 15 and means were separated (Steel and Torrie, 1980) at $P<0.05$ using Duncan New Multiple Range Test (DNMRT).

\subsection{Results and Discussion}

Heavy metals have maximum permissible level in plants specified by different bodies (Pam et al., 2014) such as FAO/WHO. Consequently, the comparison and interpretation of the results of analyzed plant samples in this study is based on the control values, standards set by FAO/WHO and the concentration of the heavy metals in soils from the study sites. The concentrations of heavy metals in plants sampled from the mined and unmined (control) sites of Akwuke and Iva in wet and dry season are presented in Tables 1 and 2, respectively. The results indicate that heavy metal concentrations differed significantly $(P<0.05)$ from one plant species to another and that the highest and the lowest metal concentrations in plants were obtained from the mined and control sites, respectively. The concentrations of $\mathrm{Ni}, \mathrm{Pb}, \mathrm{Cd}, \mathrm{Fe}$ and $\mathrm{As}$ in plants at the mined sites (Akwuke and Iva) were significantly $(P<0.05)$ higher than their corresponding values at the control site. The highest values of $\mathrm{Ni}(9.02 \pm 0.03 \mathrm{mg} / \mathrm{kg}), \mathrm{Pb}(10.37 \pm 0.52 \mathrm{mg} / \mathrm{kg})$, and $\mathrm{Fe}(231.00 \pm 1.41 \mathrm{mg} / \mathrm{kg})$ in Alchornea cordifolia, Andropogon gayanus, and Irvingia gabonensis at the site in Akwuke mine as well as the concentrations of Cd $(2.16 \pm 0.07 \mathrm{mg} / \mathrm{kg})$ and As $(0.005 \pm 0.001 \mathrm{mg} / \mathrm{kg})$ in A. cordifolia at the site in Iva mine were significantly $(P<0.05)$ higher than the highest corresponding values of $\mathrm{Ni}(0.02 \pm$ $0.01), \mathrm{Pb}(0.01 \pm 0.00 \mathrm{mg} / \mathrm{kg}), \mathrm{Fe}(1.31 \pm 0.40 \mathrm{mg} / \mathrm{kg}), \mathrm{Cd}(0.01 \pm 0.00 \mathrm{mg} / \mathrm{kg})$, and As $(0.00 \pm 0.00)$ in plants at the control site. The highest values of $\mathrm{Ni}, \mathrm{Pb}, \mathrm{Fe}, \mathrm{Cd}$, and $\mathrm{As}$ in plants obtained from the mined sites exceeded their corresponding values at the control site by 451, 1037, 176, 216, and 0.005 times, respectively. This result corroborates with the findings of Lee et al. (2001), Mtui et al. (2006) and Jung (2008) who reported that the concentration of heavy metal in plants at the mined site is higher than the concentration of plants at the control site. In the vicinity of a $\mathrm{Pb} / \mathrm{Zn}$ mine in Shaoxing, eastern China, $\mathrm{Pb}$ and $\mathrm{Cd}$ concentrations of some plants were 20 and 30 times higher than the 
permitted standards (Li et al., 2006a) while Chinese cabbage growing in the vicinity of nonferrous metals mining and smelting sites in Baiyin, China contained high concentration of $\mathrm{Cd}$ that exceeded the maximum permitted levels $(0.05 \mathrm{mg} / \mathrm{kg})$ by 4.5 times (Li et al., 2006b).

Table 1: Heavy metal concentration $(\mathrm{mg} / \mathrm{kg})$ in plants in wet and dry season at Akwuke mine site

\begin{tabular}{|c|c|c|c|c|c|}
\hline Plants & $\mathrm{Ni}$ & $\mathrm{Pb}$ & $\mathrm{Cd}$ & $\mathrm{Fe}$ & As \\
\hline Irvingia gabonensis (wet) & $4.28^{\mathrm{e}} \pm 0.10$ & $2.70^{\mathrm{e}} \pm 0.07$ & $0.97^{\mathfrak{c}} \pm 0.08$ & $2.96^{\mathrm{i}} \pm 0.08$ & $0.001^{\mathrm{de}} \pm 0.000$ \\
\hline Irvingia gabonensis (dry) & $3.06^{\mathrm{g}} \pm 0.14$ & $1.96^{\mathrm{f}} \pm 0.07$ & $0.68^{\mathrm{d}} \pm 0.03$ & $2.16^{\mathrm{j}} \pm 0.10$ & $0.000 \pm 0.000$ \\
\hline Irvingia gabonensis control (wet) & $0.01^{1} \pm 0.00$ & $0.01^{\mathrm{i}} \pm 0.00$ & $0.01^{\mathrm{i}} \pm 0.00$ & $0.27^{\mathrm{Imn}} \pm 0.04$ & $0.000 \pm 0.000$ \\
\hline Irvingia gabonensis control (dry) & $0.01^{1} \pm 0.01$ & $0.01^{\mathrm{i}} \pm 0.00$ & $0.01^{\mathrm{i}} \pm 0.01$ & $0.25^{\operatorname{lmn}} \pm 0.04$ & $0.000 \pm 0.000$ \\
\hline Dialium guinense (wet) & $3.41^{\mathrm{f}} \pm 0.16$ & $2.96^{\mathrm{d}} \pm 0.08$ & $2.02^{\mathrm{a}} \pm 0.03$ & $23.64^{\mathrm{c}} \pm 0.91$ & $0.004^{\mathrm{b}} \pm 0.001$ \\
\hline Dialium guinense (dry) & $1.98^{\mathrm{h}} \pm 0.10$ & $2.03^{f} \pm 0.06$ & $1.87^{\mathrm{b}} \pm 0.07$ & $17.81^{\mathrm{d}} \pm 0.44$ & $0.002^{\text {cd }} \pm 0.001$ \\
\hline Dialium guinense control (wet) & $0.01^{1} \pm 0.00$ & $0.01^{\mathrm{i}} \pm 0.00$ & $0.01^{\mathrm{i}} \pm 0.01$ & $1.31^{\mathrm{k}} \pm 0.40$ & $0.000 \pm 0.000$ \\
\hline Dialium guinense control (dry) & $0.01^{1} \pm 0.00$ & $0.01^{\mathrm{i}} \pm 0.01$ & $0.00^{\mathrm{i}} \pm 0.00$ & $1.28^{\mathrm{k}} \pm 0.40$ & $0.000 \pm 0.000$ \\
\hline Mangifera indica (wet) & $1.62^{\mathrm{i}} \pm 0.06$ & $0.41^{\mathrm{h}} \pm 0.03$ & $0.61^{\mathrm{e}} \pm 0.01$ & $46.00^{\mathrm{a}} \pm 1.41$ & $0.001 \pm 0.000$ \\
\hline Mangifera indica (dry) & $0.84^{\mathrm{k}} \pm 0.04$ & $0.33^{\mathrm{h}} \pm 0.03$ & $0.49^{f} \pm 0.06$ & $33.45^{\mathrm{b}} \pm 0.21$ & $0.000 \pm 0.000$ \\
\hline Mangifera indica control (wet) & $0.02^{1} \pm 0.01$ & $0.01^{\mathrm{i}} \pm 0.01$ & $0.01^{\mathrm{i}} \pm 0.01$ & $0.76^{\mathrm{klmn}} \pm 0.08$ & $0.000 \pm 0.000$ \\
\hline Mangifera indica control (dry) & $0.02^{1} \pm 0.01$ & $0.01^{\mathrm{i}} \pm 0.01$ & $0.00^{\mathrm{i}} \pm 0.00$ & $0.74^{\mathrm{lmn}} \pm 0.08$ & $0.000 \pm 0.000$ \\
\hline Icacina trichantha (wet) & $1.27^{j} \pm 0.08$ & $0.80^{\mathrm{g}} \pm 0.07$ & $0.34^{\mathrm{g}} \pm 0.06$ & $12.10^{\mathrm{f}} \pm 0.14$ & $0.001^{\mathrm{de}} \pm 0.000$ \\
\hline Icacina trichantha (dry) & $1.13^{j} \pm 0.16$ & $0.69^{\mathrm{g}} \pm 0.06$ & $0.27^{\mathrm{g}} \pm 0.03$ & $10.96^{\mathrm{g}} \pm 0.23$ & $0.001^{\mathrm{de}} \pm 0.000$ \\
\hline Icacina trichantha control (wet) & $0.01^{1} \pm 0.00$ & $0.00^{\mathrm{i}} \pm 0.00$ & $0.01^{\mathrm{i}} \pm 0.01$ & $0.08^{\mathrm{Imn}} \pm 0.02$ & $0.000 \pm 0.000$ \\
\hline Icacina trichantha control (dry) & $0.01^{1} \pm 0.00$ & $0.00^{\mathrm{i}} \pm 0.00$ & $0.01^{\mathrm{i}} \pm 0.01$ & $0.06^{\operatorname{lmn}} \pm .03$ & $0.000 \pm 0.000$ \\
\hline Alchornea cordifolia (wet) & $9.02^{\mathrm{a}} \pm 0.03$ & $3.60^{\mathrm{c}} \pm 0.14$ & $0.27^{\mathrm{g}} \pm 0.08$ & $18.04^{\mathrm{d}} \pm 0.06$ & $0.005^{\mathrm{a}} \pm 0.001$ \\
\hline Alchornea cordifolia (dry) & $5.04^{\mathrm{c}} \pm 0.07$ & $2.82^{\mathrm{de}} \pm 0.04$ & $0.16^{\mathrm{h}} \pm 0.03$ & $10.02^{\mathrm{h}} \pm 0.03$ & $0.000 \pm 0.000$ \\
\hline Alchornea cordifolia control (wet) & $0.02^{1} \pm 0.00$ & $0.01^{\mathrm{i}} \pm 0.01$ & $0.01^{\mathrm{i}} \pm 0.00$ & $0.90^{\mathrm{kl}} \pm 0.09$ & $0.000 \pm 0.000$ \\
\hline Alchornea cordifolia control (dry) & $0.02^{1} \pm 0.01$ & $0.00^{\mathrm{i}} \pm 0.00$ & $0.01^{\mathrm{i}} \pm 0.00$ & $0.87^{\mathrm{klm}} \pm 0.08$ & $0.000 \pm 0.000$ \\
\hline Andropogon gayanus (wet) & $6.42^{\mathrm{b}} \pm 0.17$ & $10.37^{\mathrm{a}} \pm 0.52$ & $0.02^{\mathrm{i}} \pm 0.01$ & $16.06^{\mathrm{e}} \pm 0.08$ & $0.000 \pm 0.000$ \\
\hline Andropogon gayanus (dry) & $4.81^{\mathrm{d}} \pm 0.04$ & $8.96^{\mathrm{b}} \pm 0.07$ & $0.01^{\mathrm{i}} \pm 0.00$ & $10.03^{\mathrm{h}} \pm 0.04$ & $0.003^{\mathrm{bc}} \pm 0.001$ \\
\hline Andropogon gayanus control (wet) & $0.01^{1} \pm 0.01$ & $0.01^{\mathrm{i}} \pm 0.00$ & $0.01^{\mathrm{i}} \pm 0.01$ & $0.39^{\operatorname{lmn}} \pm 0.11$ & $0.000 \pm 0.000$ \\
\hline Andropogon gayanus control (dry) & $0.00^{1} \pm 0.00$ & $0.01^{\mathrm{i}} \pm 0.01$ & $0.00^{\mathrm{i}} \pm 0.00$ & $0.38^{\operatorname{lmn}} \pm 0.12$ & $0.000 \pm 0.000$ \\
\hline Manihot esculenta (wet) & $0.01^{1} \pm 0.00$ & $0.01^{\mathrm{i}} \pm 0.00$ & $0.01^{\mathrm{i}} \pm 0.00$ & $0.02^{\mathrm{mn}} \pm 0.01$ & $0.000 \pm 0.000$ \\
\hline Manihot esculenta (dry) & $0.01^{1} \pm 0.00$ & $0.01^{\mathrm{i}} \pm 0.00$ & $0.01^{\mathrm{i}} \pm 0.00$ & $0.01^{\mathrm{n}} \pm 0.00$ & $0.000 \pm 0.000$ \\
\hline Manihot esculenta control (wet) & $0.02^{1} \pm 0.01$ & $0.01^{\mathrm{i}} \pm 0.01$ & $0.01^{\mathrm{i}} \pm 0.01$ & $0.14^{\operatorname{lmn}} \pm 0.01$ & $0.000 \pm 0.000$ \\
\hline Manihot esculenta control (dry) & $0.02^{1} \pm 0.01$ & $0.01^{\mathrm{i}} \pm 0.01$ & $0.01^{\mathrm{i}} \pm 0.01$ & $0.13^{\operatorname{lmn}} \pm 0.02$ & $0.000 \pm 0.000$ \\
\hline
\end{tabular}

$a, b, c, d, e, f, g, h, i, j, k, l, m, n$ means in a column with different superscript are significantly different $(P<0.05)$, values are mean \pm standard deviation of 3 replications

Table 2: Heavy metal concentration $(\mathrm{mg} / \mathrm{kg})$ in plants in wet and dry seasons at Iva mine site

\begin{tabular}{|c|c|c|c|c|c|}
\hline Plants & $\mathrm{Ni}$ & $\mathrm{Pb}$ & $\mathrm{Cd}$ & $\mathrm{Fe}$ & As \\
\hline Irvingia gabonensis (wet) & $0.43^{\mathrm{h}} \pm 0.03$ & $0.92^{\mathrm{i}} \pm 0.03$ & $0.74^{\mathrm{f}} \pm 0.04$ & $231.00^{\mathrm{a}} \pm 1.41$ & $0.000 \pm 0.000$ \\
\hline Irvingia gabonensis (dry) & $0.35^{\mathrm{h}} \pm 0.06$ & $0.81^{\mathrm{j}} \pm 0.06$ & $0.62^{\mathrm{g}} \pm 0.03$ & $217.00^{\mathrm{b}} \pm 1.56$ & $0.000 \pm 0.000$ \\
\hline Irvingia gabonensis control (wet) & $0.01^{\mathrm{i}} \pm 0.00$ & $0.01^{\mathrm{k}} \pm 0.00$ & $0.01^{\mathrm{i}} \pm 0.00$ & $0.27^{\mathrm{m}} \pm 0.04$ & $0.000 \pm 0.000$ \\
\hline Irvingia gabonensis control (dry) & $0.01^{\mathrm{i}} \pm 0.01$ & $0.01^{\mathrm{k}} \pm 0.00$ & $0.01^{\mathrm{i}} \pm 0.01$ & $0.25^{\mathrm{m}} \pm 0.04$ & $0.000 \pm 0.000$ \\
\hline Dialium guinense (wet) & $2.07^{\mathrm{C}} \pm 0.04$ & $3.30^{\mathrm{b}} \pm 0.14$ & $0.62^{g} \pm 0.03$ & $80.60^{\mathrm{i}} \pm 0.85$ & $0.001^{\mathrm{bc}} \pm 0.000$ \\
\hline Dialium guinense (dry) & $1.69^{\mathrm{d}} \pm 0.08$ & $3.11^{\mathrm{c}} \pm 0.03$ & $0.39^{\mathrm{h}} \pm 0.04$ & $69.80^{\mathrm{j}} \pm 1.13$ & $0.000 \pm 0.000$ \\
\hline Dialium guinense control (wet) & $0.01^{\mathrm{i}} \pm 0.00$ & $0.01^{\mathrm{k}} \pm 0.00$ & $0.01^{\mathrm{i}} \pm 0.01$ & $1.31^{\mathrm{m}} \pm 0.40$ & $0.000 \pm 0.000$ \\
\hline Dialium guinense control (dry) & $0.01^{\mathrm{i}} \pm 0.00$ & $0.01^{\mathrm{k}} \pm 0.01$ & $0.00^{\mathrm{i}} \pm 0.00$ & $1.28^{\mathrm{m}} \pm 0.40$ & $0.000 \pm 0.000$ \\
\hline Mangifera indica (wet) & $1.31^{\mathrm{e}} \pm 0.03$ & $3.69^{\mathrm{a}} \pm 0.06$ & $1.06^{\mathrm{e}} \pm 0.08$ & $153.50^{c} \pm 0.71$ & $0.003^{\mathrm{a}} \pm 0.001$ \\
\hline Mangifera indica (dry) & $1.01^{\mathrm{g}} \pm 0.03$ & $2.87^{\mathrm{d}} \pm 0.08$ & $1.01^{\mathrm{e}} \pm 0.04$ & $138.20^{\mathrm{d}} \pm 0.42$ & $0.001^{\mathrm{bc}} \pm 0.000$ \\
\hline Mangifera indica control (wet) & $0.02^{\mathrm{i}} \pm 0.01$ & $0.01^{\mathrm{k}} \pm 0.01$ & $0.01^{\mathrm{i}} \pm 0.01$ & $0.76^{\mathrm{m}} \pm 0.08$ & $0.000 \pm 0.000$ \\
\hline Mangifera indica control (dry) & $0.02^{\mathrm{i}} \pm 0.01$ & $0.01^{\mathrm{k}} \pm 0.01$ & $0.00^{\mathrm{i}} \pm 0.00$ & $0.74^{\mathrm{m}} \pm 0.08$ & $0.000 \pm 0.000$ \\
\hline Icacina trichantha (wet) & $4.10^{\mathrm{a}} \pm 0.14$ & $1.46^{\mathrm{g}} \pm 0.01$ & $1.38^{\mathrm{c}} \pm 0.04$ & $128.70^{\mathrm{e}} \pm 0.99$ & $0.002^{\mathrm{b}} \pm 0.001$ \\
\hline Icacina trichantha (dry) & $3.48^{\mathrm{b}} \pm 0.13$ & $1.03^{\mathrm{h}} \pm 0.06$ & $1.19^{\mathrm{d}} \pm 0.13$ & $103.10^{\mathrm{g}} \pm 0.42$ & $0.001^{\mathrm{bc}} \pm 0.000$ \\
\hline Icacina trichantha control (wet) & $0.01^{\mathrm{i}} \pm 0.00$ & $0.00^{\mathrm{k}} \pm 0.00$ & $0.01^{\mathrm{i}} \pm 0.01$ & $0.08^{\mathrm{m}} \pm 0.02$ & $0.000 \pm 0.000$ \\
\hline Icacina trichantha control (dry) & $0.01^{\mathrm{i}} \pm 0.00$ & $0.00^{\mathrm{k}} \pm 0.00$ & $0.01^{\mathrm{i}} \pm 0.01$ & $0.06^{\mathrm{m}} \pm .03$ & $0.000 \pm 0.000$ \\
\hline Alchornea cordifolia (wet) & $1.37^{\mathrm{e}} \pm 0.08$ & $2.73^{\mathrm{e}} \pm 0.03$ & $2.16^{\mathrm{a}} \pm 0.07$ & $108.20^{\mathrm{f}} \pm 0.28$ & $0.001^{\mathrm{bc}} \pm 0.000$ \\
\hline Alchornea cordifolia (dry) & $1.13^{\mathrm{f}} \pm 0.16$ & $2.03^{\mathrm{f}} \pm 0.04$ & $1.90^{\mathrm{b}} \pm 0.03$ & $83.80^{\mathrm{h}} \pm 1.27$ & $0.000 \pm 0.000$ \\
\hline Alchornea cordifolia control (wet) & $0.02^{\mathrm{i}} \pm 0.00$ & $0.01^{\mathrm{k}} \pm 0.01$ & $0.01^{\mathrm{i}} \pm 0.00$ & $0.90^{\mathrm{m}} \pm 0.09$ & $0.000 \pm 0.000$ \\
\hline Alchornea cordifolia control (dry) & $0.02^{\mathrm{i}} \pm 0.01$ & $0.00^{\mathrm{k}} \pm 0.00$ & $0.01^{\mathrm{i}} \pm 0.00$ & $0.87^{\mathrm{m}} \pm 0.08$ & $0.000 \pm 0.000$ \\
\hline Andropogon gayanus (wet) & $0.10^{\mathrm{i}} \pm 0.01$ & $0.06^{\mathrm{k}} \pm 0.01$ & $0.06^{\mathrm{i}} \pm 0.01$ & $68.00^{\mathrm{k}} \pm 0.71$ & $0.001^{\mathrm{bc}} \pm 0.001$ \\
\hline Andropogon gayanus (dry) & $0.07^{\mathrm{i}} \pm 0.03$ & $0.05^{\mathrm{k}} \pm 0.01$ & $0.05^{\mathrm{i}} \pm 0.01$ & $59.70^{1} \pm 1.27$ & $0.001^{\mathrm{bc}} \pm 0.000$ \\
\hline Andropogon gayanus control (wet) & $0.01^{\mathrm{i}} \pm 0.01$ & $0.01^{\mathrm{k}} \pm 0.00$ & $0.01^{\mathrm{i}} \pm 0.01$ & $0.39^{\mathrm{m}} \pm 0.11$ & $0.000 \pm 0.000$ \\
\hline Andropogon gayanus control (dry) & $0.00^{\mathrm{i}} \pm 0.00$ & $0.01^{\mathrm{k}} \pm 0.01$ & $0.00^{\mathrm{i}} \pm 0.00$ & $0.38^{\mathrm{m}} \pm 0.12$ & $0.000 \pm 0.000$ \\
\hline Manihot esculenta (wet) & $0.01^{\mathrm{i}} \pm 0.00$ & $0.01^{\mathrm{k}} \pm 0.00$ & $0.01 \pm 0.00$ & $0.02^{\mathrm{m}} \pm 0.01$ & $0.000 \pm 0.000$ \\
\hline Manihot esculenta (dry) & $0.01^{\mathrm{i}} \pm 0.00$ & $0.01^{\mathrm{k}} \pm 0.00$ & $0.01^{\mathrm{i}} \pm 0.00$ & $0.01^{\mathrm{m}} \pm 0.00$ & $0.000 \pm 0.000$ \\
\hline Manihot esculenta control (wet) & $0.02^{\mathrm{i}} \pm 0.01$ & $0.01^{\mathrm{k}} \pm 0.01$ & $0.01^{\mathrm{i}} \pm 0.01$ & $0.14^{\mathrm{m}} \pm 0.01$ & $0.000 \pm 0.000$ \\
\hline Manihot esculenta control (dry) & $0.02^{\mathrm{i}} \pm 0.01$ & $0.01^{\mathrm{k}} \pm 0.01$ & $0.01^{\mathrm{i}} \pm 0.01$ & $0.13^{\mathrm{m}} \pm 0.02$ & $0.000 \pm 0.000$ \\
\hline
\end{tabular}

$a, b, c, d, e, f, g, h, i, j, k, l, m, n, o, p, q, r, s, t$, means in a column with different superscript are significantly different $(P<0.05)$, values are mean \pm

standard deviation of 3 replications 
The high concentration of heavy metals in plant species sampled from the mined sites may be attributed to pollutants generated during mining activities. Mining activities such as mineral excavation, ore transportation, smelting and refining, and disposal of tailings and waste waters around mines are important sources of heavy metals build up in plants and phytotoxicity in the environment (Dudka and Adriano, 1997; Pruvot et al., 2006; Perez-Sirvent et al., 2008; Ogbonna et al., 2018a). Since there was no other visible sources of contamination around the study area, the high concentrations of heavy metals in soil (Tables 3 and 4) at the two mined sites are implicated for the higher metal concentrations in plants. Several studies in China, South Korea, and the United States of America have shown that plants are often contaminated by heavy metals dispersed from mining and smelting operations (Chang et al., 2005; Yang et al., 2006; Zheng et al., 2007).

On a comparative basis of plants investigated in this study, the concentration of $\mathrm{Ni}$, As, and $\mathrm{Cd}$ was highest in A. cordifolia. The high concentration of these metals (Ni, As, and $\mathrm{Cd}$ ) in A. cordifolia may be attributed to inherent ability of the plant (A. cordifolia) to absorb and translocate more $\mathrm{Ni}$, As, and $\mathrm{Cd}$ to the aerial plant parts (leaves) than other plants. Some plants can tolerate high heavy metals concentration from soil (McGrath et al., 2001) by binding metals to cell walls, compartmentalizing them in vacuoles or complexing them to certain organic acids or proteins (Reeves and Baker, 2000). Similarly, heavy metals in soils exist in variable forms, such as exchangeable or absorbed forms, which influence their mobility and bioavailability for uptake by plants and transfer to the aerial parts of plants (Ogbonna et al., 2012). Indeed, the concentration of Pb was highest $(10.37 \pm 0.52 \mathrm{mg} / \mathrm{kg}$ ) in A. gayanus while Fe concentration was highest $(231.00 \pm 1.41 \mathrm{mg} / \mathrm{kg})$ in I. gabonensis. Plant species differ in their ability to take up, accumulate and tolerate heavy metals and this variation occurs not only among plant species but also within a given species (Raskin et al., 1997; Angelova et al., 2004). Since $\mathrm{Pb}$ concentration in soils was higher at Iva mine, the higher concentration of $\mathrm{Pb}$ in A. gayanus at Akwuke mine may be attributed to inherent ability of the plant to take up $\mathrm{Pb}$ from the soil and translocate more to the leaves than other plants at the mined sites. The rate of metal movement in plant tissues varies depending on plant organ, age and element involved (Kabata-Pendias, 2000).

Table 3: Heavy metal concentration $(\mathrm{mg} / \mathrm{kg})$ in soil at Iva mine in wet and dry season

\begin{tabular}{|c|c|c|c|c|c|c|c|}
\hline Location & Depth & Season & $\mathrm{Ni}$ & $\mathrm{Pb}$ & As & $\mathrm{Fe}$ & $\mathrm{Cd}$ \\
\hline \multirow{6}{*}{ Crest } & \multirow{2}{*}{$0-10 \mathrm{~cm}$} & Wet & $28.01^{\mathrm{g}} \pm 0.03$ & $55.70^{\mathrm{d}} \pm 1.13$ & $0.00 \pm 0.00$ & $377.30^{j} \pm 3.82$ & $5.02^{\mathrm{a}} \pm 0.11$ \\
\hline & & Dry & $20.05^{\mathrm{i}} \pm 0.21$ & $48.25^{\mathrm{e}} \pm 0.21$ & $0.00 \pm 0.00$ & $353.00^{\mathrm{k}} \pm 1.27$ & $0.11^{\mathrm{kl}} \pm 0.03$ \\
\hline & \multirow{2}{*}{$10-20 \mathrm{~cm}$} & Wet & $15.25^{j} \pm 0.42$ & $45.30^{f} \pm 0.57$ & $0.00 \pm 0.00$ & $387.50^{\mathrm{i}} \pm 3.54$ & $2.87^{\mathrm{C}} \pm 0.13$ \\
\hline & & Dry & $10.05^{\mathrm{k}} \pm 0.78$ & $22.05^{\mathrm{m}} \pm 0.35$ & $0.00 \pm 0.00$ & $238.60^{\mathrm{n}} \pm 0.28$ & $0.07^{\mathrm{kl}} \pm 0.03$ \\
\hline & \multirow{2}{*}{$20-30 \mathrm{~cm}$} & Wet & $10.00^{\mathrm{k}} \pm 0.57$ & $38.10^{\mathrm{h}} \pm 0.28$ & $0.00 \pm 0.00$ & $402.80^{\mathrm{fg}} \pm 2.55$ & $1.01^{\mathrm{g}} \pm 0.13$ \\
\hline & & Dry & $6.10^{1} \pm 0.42$ & $29.40^{j} \pm 0.85$ & $0.00 \pm 0.00$ & $218.00^{\circ} \pm 2.83$ & $0.48^{\mathrm{ij}} \pm 0.13$ \\
\hline \multirow{6}{*}{ Middle slope } & \multirow{2}{*}{$0-10 \mathrm{~cm}$} & Wet & $46.45^{\mathrm{d}} \pm 0.16$ & $37.50^{\mathrm{hi}} \pm 0.28$ & $0.00 \pm 0.00$ & $410.30^{\mathrm{e}} \pm 2.40$ & $0.49^{\mathrm{ij}} \pm 0.08$ \\
\hline & & Dry & $21.05^{\mathrm{i}} \pm 1.34$ & $30.01^{\mathrm{j}} \pm 1.40$ & $0.00 \pm 0.00$ & $238.60^{\mathrm{n}} \pm 1.56$ & $0.19^{\mathrm{k}} \pm 0.01$ \\
\hline & \multirow{2}{*}{$10-20 \mathrm{~cm}$} & Wet & $64.12^{b} \pm 0.35$ & $65.04^{\mathrm{c}} \pm 0.34$ & $0.00 \pm 0.00$ & $467.80^{c} \pm 3.11$ & $1.64^{\mathrm{e}} \pm 0.07$ \\
\hline & & Dry & $40.06^{\mathrm{e}} \pm 1.33$ & $43.60^{g} \pm 1.56$ & $0.00 \pm 0.00$ & $392.70^{\text {hi }} \pm 3.82$ & $0.04^{\mathrm{kl}} \pm 0.03$ \\
\hline & \multirow{2}{*}{$20-30 \mathrm{~cm}$} & Wet & $79.00^{\mathrm{a}} \pm 0.57$ & $81.60^{\mathrm{a}} \pm 0.57$ & $0.00 \pm 0.00$ & $498.20^{\mathrm{a}} \pm 2.55$ & $1.86^{\mathrm{d}} \pm 0.23$ \\
\hline & & Dry & $52.30^{c} \pm 0.57$ & $69.70^{\mathrm{b}} \pm 2.40$ & $0.00 \pm 0.00$ & $408.00^{\mathrm{ef}} \pm 2.83$ & $1.17^{\mathrm{f}} \pm 0.04$ \\
\hline \multirow{6}{*}{ Valley } & \multirow{2}{*}{$0-10 \mathrm{~cm}$} & Wet & $15.20^{j} \pm 0.57$ & $36.65^{\mathrm{hi}} \pm 0.35$ & $0.00 \pm 0.00$ & $328.50^{1} \pm 2.12$ & $3.08^{b} \pm 0.03$ \\
\hline & & Dry & $10.06^{k} \pm 0.65$ & $23.85^{1} \pm 0.21$ & $0.00 \pm 0.00$ & $397.70^{\text {gh }} \pm 0.42$ & $0.01^{\mathrm{kl}} \pm 0.01$ \\
\hline & \multirow{2}{*}{$10-20 \mathrm{~cm}$} & Wet & $23.50^{\mathrm{h}} \pm 0.37$ & $35.90^{i} \pm 0.85$ & $0.00 \pm 0.00$ & $454.70^{\mathrm{d}} \pm 0.42$ & $0.74^{\mathrm{h}} \pm 0.07$ \\
\hline & & Dry & $10.05^{\mathrm{k}} \pm 0.49$ & $27.01^{\mathrm{k}} \pm 0.01$ & $0.00 \pm 0.00$ & $273.70^{\mathrm{m}} \pm 0.42$ & $0.08^{\mathrm{kl}} \pm 0.03$ \\
\hline & \multirow{2}{*}{$20-30 \mathrm{~cm}$} & Wet & $31.07^{\mathrm{f}} \pm 0.38$ & $26.10^{k} \pm 0.28$ & $0.00 \pm 0.00$ & $490.00^{b} \pm 5.66$ & $0.61^{\text {hi }} \pm 0.04$ \\
\hline & & Dry & $23.13^{\mathrm{h}} \pm 0.33$ & $19.60^{\mathrm{n}} \pm 0.28$ & $0.00 \pm 0.00$ & $381.40^{\mathrm{j}} \pm 1.98$ & $0.41^{\mathrm{j}} \pm 0.07$ \\
\hline \multirow{6}{*}{ Control } & \multirow{2}{*}{$0-10 \mathrm{~cm}$} & Wet & $0.02^{\mathrm{m}} \pm 0.01$ & $1.48^{\circ} \pm 0.12$ & $0.00 \pm 0.00$ & $70.60^{r} \pm 0.57$ & $0.01^{\mathrm{kl}} \pm 0.00$ \\
\hline & & Dry & $0.02^{\mathrm{m}} \pm 0.01$ & $0.72^{\circ} \pm 0.04$ & $0.00 \pm 0.00$ & $43.00^{t} \pm 0.57$ & $0.00^{1} \pm 0.00$ \\
\hline & \multirow{2}{*}{$10-20 \mathrm{~cm}$} & Wet & $0.01^{\mathrm{m}} \pm 0.00$ & $1.01^{\circ} \pm 0.16$ & $0.00 \pm 0.00$ & $82.00^{\mathrm{q}} \pm 0.71$ & $0.00^{1} \pm 0.00$ \\
\hline & & Dry & $0.00^{\mathrm{m}} \pm 0.00$ & $0.40^{\circ} \pm 0.06$ & $0.00 \pm 0.00$ & $38.50^{t} \pm 0.85$ & $0.00^{1} \pm 0.00$ \\
\hline & \multirow{2}{*}{$20-30 \mathrm{~cm}$} & Wet & $0.01^{\mathrm{m}} \pm 0.01$ & $0.26^{\circ} \pm 0.10$ & $0.00 \pm 0.00$ & $101.00^{\mathrm{p}} \pm 1.41$ & $0.00^{1} \pm 0.00$ \\
\hline & & Dry & $0.00^{\mathrm{m}} \pm 0.00$ & $0.22^{\circ} \pm 0.08$ & $0.00 \pm 0.00$ & $62.00^{\mathrm{s}} \pm 4.24$ & $0.00^{1} \pm 0.00$ \\
\hline
\end{tabular}

Source: Ogbonna et al. (2018a) 
Table 4: Heavy metal concentration $(\mathrm{mg} / \mathrm{kg})$ in soil at Akwuke mine site in wet and dry season

\begin{tabular}{|c|c|c|c|c|c|c|c|}
\hline Location & Depth $(\mathrm{cm})$ & Season & $\mathrm{Ni}$ & $\mathrm{Pb}$ & As & $\mathrm{Fe}$ & $\mathrm{Cd}$ \\
\hline \multirow{6}{*}{ Crest } & \multirow{2}{*}{$0-10$} & Rainy & $9.71^{\mathrm{efg}} \pm 0.16$ & $15.45^{\mathrm{bc}} \pm 0.18$ & $0.00 \pm 0.00$ & $417.00^{\mathrm{f}} \pm 1.98$ & $1.73^{\mathrm{c}} \pm 0.06$ \\
\hline & & Dry & $4.15^{\mathrm{k}} \pm 0.07$ & $12.65^{\mathrm{cd}} \pm 0.13$ & $0.00 \pm 0.00$ & $296.10^{\mathrm{k}} \pm 8.63$ & $0.68^{\mathrm{de}} \pm 0.07$ \\
\hline & \multirow{2}{*}{$10-20$} & Rainy & $11.72^{\mathrm{d}} \pm 0.07$ & $15.80^{\mathrm{bc}} \pm 0.28$ & $0.00 \pm 0.00$ & $480.70^{\mathrm{c}} \pm 2.40$ & $0.72^{\mathrm{d}} \pm 0.17$ \\
\hline & & Dry & $10.04^{\mathrm{ef}} \pm 0.20$ & $10.45^{\mathrm{de}} \pm 0.07$ & $0.00 \pm 0.00$ & $290.00^{\mathrm{k}} \pm 4.95$ & $0.07^{\mathrm{gh}} \pm 0.03$ \\
\hline & \multirow{2}{*}{$20-30$} & Rainy & $14.30^{\mathrm{c}} \pm 0.71$ & $17.62^{\mathrm{b}} \pm 0.08$ & $0.00 \pm 0.00$ & $522.00^{\mathrm{a}} \pm 1.98$ & $0.61^{\mathrm{de}} \pm 0.13$ \\
\hline & & Dry & $8.24^{\mathrm{hi}} \pm 0.20$ & $13.60^{\mathrm{cd}} \pm 0.28$ & $0.00 \pm 0.00$ & $216.40^{\mathrm{n}} \pm 1.27$ & $0.38^{\mathrm{f}} \pm 0.03$ \\
\hline \multirow{6}{*}{ Middle slope } & \multirow{2}{*}{$0-10$} & Rainy & $10.45^{\mathrm{e}} \pm 0.27$ & $15.10^{\mathrm{bc}} \pm 0.28$ & $0.00 \pm 0.00$ & $428.10^{\mathrm{e}} \pm 0.85$ & $2.92^{\mathrm{b}} \pm 0.03$ \\
\hline & & Dry & $6.04^{j} \pm 0.06$ & $10.41^{\mathrm{de}} \pm 0.11$ & $0.00 \pm 0.00$ & $362.20^{i} \pm 4.53$ & $0.10^{\mathrm{gh}} \pm 0.04$ \\
\hline & \multirow{2}{*}{$10-20$} & Rainy & $11.65^{\mathrm{d}} \pm 0.11$ & $12.60^{\mathrm{cd}} \pm 0.35$ & $0.00 \pm 0.00$ & $458.00^{\mathrm{d}} \pm 2.12$ & $0.17^{\mathrm{g}} \pm 0.06$ \\
\hline & & Dry & $8.04^{i} \pm 0.20$ & $8.21^{\mathrm{ef}} \pm 0.44$ & $0.00 \pm 0.00$ & $267.60^{1} \pm 1.56$ & $0.01^{\mathrm{h}} \pm 0.01$ \\
\hline & \multirow{2}{*}{$20-30$} & Rainy & $12.02^{\mathrm{d}} \pm 0.48$ & $10.30^{\mathrm{de}} \pm 0.57$ & $0.00 \pm 0.00$ & $506.70^{\mathrm{b}} \pm 0.42$ & $0.08^{\mathrm{gh}} \pm 0.01$ \\
\hline & & Dry & $9.20^{\mathrm{fgh}} \pm 0.42$ & $6.11^{f} \pm 0.13$ & $0.00 \pm 0.00$ & $314.00^{j} \pm 1.56$ & $0.02^{\mathrm{h}} \pm 0.01$ \\
\hline \multirow{6}{*}{ Valley } & \multirow{2}{*}{$0-10$} & Rainy & $8.85^{\text {ghi }} \pm 0.35$ & $15.26^{\mathrm{bc}} \pm 6.87$ & $0.00 \pm 0.00$ & $397.80^{g} \pm 11.03$ & $3.06^{\mathrm{a}} \pm 0.08$ \\
\hline & & Dry & $6.07^{\mathrm{j}} \pm 0.10$ & $17.50^{b} \pm 0.42$ & $0.00 \pm 0.00$ & $389.80^{\mathrm{h}} \pm 0.28$ & $0.08^{\mathrm{gh}} \pm 0.03$ \\
\hline & \multirow{2}{*}{$10-20$} & Rainy & $16.03^{b} \pm 0.21$ & $21.10^{a} \pm 0.85$ & $0.00 \pm 0.00$ & $258.70^{\mathrm{m}} \pm 0.42$ & $0.57^{\mathrm{e}} \pm 0.10$ \\
\hline & & Dry & $10.04^{\mathrm{ef}} \pm 1.47$ & $15.65^{\mathrm{bc}} \pm 0.35$ & $0.00 \pm 0.00$ & $207.80^{\circ} \pm 3.11$ & $0.05^{\mathrm{gh}} \pm 0.03$ \\
\hline & \multirow{2}{*}{$20-30$} & Rainy & $19.81^{\mathrm{a}} \pm 1.29$ & $21.72^{\mathrm{a}} \pm 0.69$ & $0.00 \pm 0.00$ & $203.00^{\circ} \pm 4.24$ & $0.29^{\mathrm{f}} \pm 0.01$ \\
\hline & & Dry & $12.01^{\mathrm{d}} \pm 0.16$ & $13.28^{\mathrm{cd}} \pm 0.11$ & $0.00 \pm 0.00$ & $186.00^{\mathrm{p}} \pm 2.83$ & $0.11^{\mathrm{gh}} \pm 0.03$ \\
\hline \multirow{6}{*}{ Control } & \multirow{2}{*}{$0-10$} & Rainy & $0.02^{1} \pm 0.00$ & $1.46^{\mathrm{g}} \pm 0.23$ & $0.00 \pm 0.00$ & $70.60^{\mathrm{s}} \pm 1.98$ & $0.01^{\mathrm{h}} \pm 0.00$ \\
\hline & & Dry & $0.02^{1} \pm 0.00$ & $0.72^{\mathrm{g}} \pm 0.04$ & $0.00 \pm 0.00$ & $43.00^{\mathrm{u}} \pm 0.99$ & $0.00^{\mathrm{h}} \pm 0.00$ \\
\hline & \multirow{2}{*}{$10-20$} & Rainy & $0.01^{1} \pm 0.00$ & $1.01^{\mathrm{g}} \pm 0.16$ & $0.00 \pm 0.00$ & $82.00^{r} \pm 2.83$ & $0.00^{\mathrm{h}} \pm 0.00$ \\
\hline & & Dry & $0.00^{1} \pm 0.00$ & $0.40^{\mathrm{g}} \pm 0.04$ & $0.00 \pm 0.00$ & $38.50^{u} \pm 0.99$ & $0.00^{\mathrm{h}} \pm 0.00$ \\
\hline & \multirow{2}{*}{$20-30$} & Rainy & $0.01^{1} \pm 0.01$ & $0.26^{\mathrm{g}} \pm 0.04$ & $0.00 \pm 0.00$ & $101.00^{\mathrm{q}} \pm 4.24$ & $0.00^{\mathrm{h}} \pm 0.00$ \\
\hline & & Dry & $0.00^{1} \pm 0.00$ & $0.22^{g} \pm 0.14$ & $0.00 \pm 0.00$ & $62.20^{t} \pm 1.41$ & $0.00^{\mathrm{h}} \pm 0.00$ \\
\hline
\end{tabular}

Khan et al. (2008) reported that lead causes both acute and chronic poisoning and thus, poses adverse effects on kidney, liver, vascular and immune system. Among the entire metallic element investigated in plants at the two mined sites, Fe (a trace metal) recorded the highest metal concentration in plants in Irvingia gabonensis $(231.00 \pm 1.41 \mathrm{mg} / \mathrm{kg})$ in wet season at Iva mine (Table 2). The high concentration of Fe in soil $(522.00 \pm 1.98 \mathrm{mg} / \mathrm{kg}$ ) at Akwuke mine (Table 3) did not culminate to high Fe concentration in plants at Akwuke mine. Consequently, heavy metal uptake via the roots from contaminated soil and direct deposition of metals from tailing dust onto plant surfaces (leaves) may have accounted for the high concentration of $\mathrm{Fe}$ in I. gabonensis at Iva mine. Iron is essential for the synthesis of chlorophyll and activates a number of respiratory enzymes in plants but its $(\mathrm{Fe})$ deficiency results in severe chlorosis of leaves in plants (Pam et al., 2014). High levels of exposure to Fe dust may cause respiratory diseases such as chronic bronchitis and ventilation difficulties. Meanwhile, arsenic had the lowest heavy metal concentration in plant, which can be associated with its (As) level in soil.

In comparing the concentration of heavy metals in soil with the concentration in plants, the results indicate that the concentration of metals were higher in soil than in plants. The highest concentration of $\mathrm{Ni}, \mathrm{Pb}, \mathrm{Fe}$, and $\mathrm{Cd}$ in soils (Tables 3 and 4$)$ at the mined sites were significantly $(P<0.05)$ higher than their highest corresponding values in plants (Tables 1 and 2 ) in this study. Thus, the concentration of $\mathrm{Fe}(522.00 \pm 1.98 \mathrm{mg} / \mathrm{kg}), \mathrm{Ni}(79.00 \pm 0.57 \mathrm{mg} / \mathrm{kg}), \mathrm{Pb}(81.60 \pm 0.57 \mathrm{mg} / \mathrm{kg})$, and Cd $(5.02 \pm 0.11 \mathrm{mg} / \mathrm{kg})$ in soil were $2.3,8.8,7.9$, and 2.3 times higher than their corresponding values in plant species, respectively. In contrast, As had higher concentration in plant than in soil. The highest concentration of As $(0.005 \mathrm{mg} / \mathrm{kg})$ in plants was 0.005 times higher than its corresponding value in soil. Consequently, the soil is implicated for the concentration of $\mathrm{Ni}, \mathrm{Pb}, \mathrm{Fe}$, and $\mathrm{Cd}$ in plants while aerial deposition may be responsible for the higher concentration of As in plants.

The concentrations of all metals in the plants were raised to different degrees in wet and dry season at the study sites. In this study, the concentrations of metals in plants were significantly $(P<0.05)$ higher in wet season than in dry season at the 2 mined (Akwuke and Iva) sites. At the site at Akwuke mine, the concentration of $\mathrm{Ni}(9.02 \pm 0.03 \mathrm{mg} / \mathrm{kg})$ and $\mathrm{As}(0.005 \pm 0.00 \mathrm{mg} / \mathrm{kg})$ observed in $A$. cordifolia, $\mathrm{Pb}(10.37 \pm 0.32 \mathrm{mg} / \mathrm{kg})$ in A. gayanus, $\mathrm{Cd}(2.02 \pm 0.03 \mathrm{mg} / \mathrm{kg})$ in D. guinense, and $\mathrm{Fe}$ $(46.00 \pm 1.41 \mathrm{mg} / \mathrm{kg})$ in $M$. indica in wet season were significantly $(P<0.05)$ higher than their corresponding values $(5.04 \pm 0.07,8.96 \pm 0.01$ and $0.003 \pm 0.001 \mathrm{mg} / \mathrm{kg}, 1.87 \pm 0.07 \mathrm{mg} / \mathrm{kg}$, and $33.45 \pm 0.21 \mathrm{mg} / \mathrm{kg}$ ) in dry season and the concentrations of $\mathrm{Ni}, \mathrm{Pb}, \mathrm{Cd}, \mathrm{Fe}$, and As in wet season at the site in Akwuke mine were 1.8, 1.2, 1.1, 1.4, and 1.7 times higher than their corresponding values in dry season, respectively. At the site of Iva mine, the concentration of Ni $(4.10 \pm 0.14 \mathrm{mg} / \mathrm{kg})$ in $I$. trichantha, $\mathrm{Pb}(3.69 \pm 0.06 \mathrm{mg} / \mathrm{kg})$ in $M$. indica, $\mathrm{Cd}(2.16 \pm 0.07 \mathrm{mg} / \mathrm{kg})$ in A. cordifolia, $\mathrm{Fe}(231 \pm$ 
$1.41 \mathrm{mg} / \mathrm{kg})$ in $I$. gabonensis, and As $(0.003 \pm 0.001 \mathrm{mg} / \mathrm{kg})$ in $M$. indica in wet season were significantly $(P<0.05)$ higher than their corresponding values $(3.48 \pm 0.13,3.11 \pm 0.08,1.90 \pm 0.03$, $217.00 \pm 1.56$, and $0.001 \pm 0.000 \mathrm{mg} / \mathrm{kg}$ ) in dry season and the concentrations of $\mathrm{Ni}, \mathrm{Pb}, \mathrm{Cd}, \mathrm{Fe}$, and As in wet season at the site in Iva mine was 1.2, 1.2, 1.1, 1.1, and 3 times higher than their corresponding values in dry season, respectively.

Different metals and different plant species show different patterns of seasonal growth and metal accumulation (Pokethitiyook et al., 2008). For instance, shoots of Spartina alteriflora accumulated $\mathrm{Mn}, \mathrm{Cu}$, and $\mathrm{Zn}$ rapidly in the spring, and then the levels decreased (Gleason et al., 1979). The decrease was attributed to a growth-dilution effect due to growth increase, whereby changes in the amount of plant biomass bring about corresponding changes in plant metal content (Deram et al., 2006). Nickel in plants ranged from $0.01 \pm 0.00-9.02 \pm 0.03 \mathrm{mg} / \mathrm{kg}$, which is higher than $0.02-5$ $\mathrm{mg} / \mathrm{kg}$ (Alloway and Ayres, 1997). Classic symptoms of Ni toxicity include interveinal chlorosis and development of perpendicular white strips on the above ground biomass (Hunter and Vergnano, 1952; Singh et al., 2010). Lead (Pb) in plants ranged from $0.01 \pm 0.00-10.37 \pm 0.52 \mathrm{mg} / \mathrm{kg}$, which is lower than $0.3-16.6 \mathrm{mg} / \mathrm{kg}$ of $\mathrm{Pb}$ observed in soybean leaves, red peppers and rice stalks and leaves at the Daduk Au-Ag-Pb-Zn mine, Korea (Lee et al., 2001), 2.90-89.00 mg/kg observed in Pergularia tomentosa, Calotropis procera, Acacia tortilis, Ochradenus baccatus, Salsola sp., Rhiza strica, Convolvulus sp., Euculeprus sp., Family graminaea, and Prosopis juliflora in Mahad AD'Dahab mine, Saudi Arabia (Al-Farraj and Al-Wabel, 2007), 56.00-160 mg/kg observed in Salsola kali, Andropogon barbinodis, Aster tanacetifolius, Baphia absinthifolia, Asphodelus sp., Solanum elaeagnifolium, and Dyssodia papposa in Zacatecas mine, Mexico (González and González-Chávez, 2006), $33.00 \pm 28.00-8967 \pm 6181 \mathrm{mg} / \mathrm{kg}$ of Pb observed in Alchornea conyzoides, Buddleja asiatica, Conyza sumatrensis, Equisetum debile, Imperata cylindica, Mimosa pudica, Neyraudia reynaudiana, Paspalum conjugatum, Phragmites karka, Sonchus arvensis, Thysanolaena maxima and Vigna umbellate at Bo Ngam lead mine, Thailand (Pokethitiyook et al., 2008) and 3.4-920 mg/kg of Pb in different wetland plant species collected from mine tailings (Stoltz and Greger, 2002). Lead is a nonessential element in metabolic processes (Alloway, 1995) and may become toxic to ecological systems, even at trace levels (Borg and Johansson, 1989; Ogbonna and Okeke, 2011). It (Pb) produced a phytotoxic impact resembling necrosis, stunted growth of roots and shoots, chlorosis, and decreased biomass production in Vetiveria zizanoides and Nicotiana tabacum (Boonyapookana et al., 2005). In man, $\mathrm{Pb}$ poisoning causes inhibition of synthesis of haemoglobin; cardiovascular system and acute and chronic damage to the central nervous system and peripheral nervous system (Ogwuegbu and Muhanga, 2005), poor development of grey matter in the brain of children, resulting in poor intelligent quotient (Udedi, 2003).

Lead $(\mathrm{Pb})$ and nickel $(\mathrm{Ni})$ concentration in Andropogon gayanus $(10.37 \pm 0.52 \mathrm{mg} / \mathrm{kg}$ ) and Alchornea cordifolia $(9.02 \pm 0.03 \mathrm{mg} / \mathrm{kg}$ ) is well above the permissible limit (PL) of $0.30 \mathrm{mg} / \mathrm{kg}$ of Pb and 1.63 $\mathrm{mg} / \mathrm{kg}$ of Ni for plants established by Codex Alimentarius Commission FAO/WHO (2007), shown in Table 5. The leaves of A. gayanus and A. cordifolia are very important forage material for West African Dwarf (WAD) goats in south-eastern Nigeria (Ogbonna et al., 2012). Thus, such $\mathrm{Pb}$ and Ni concentrations in plant aerial parts might be detrimental for the local herbivorous fauna, which could be unable to detect the presence of toxic metals in plant tissues as shown for snails (Noret et al., 2007). The concentration of $\mathrm{Cd}$ in plants ranged from $0.01 \pm 0.00-2.16 \pm 0.07 \mathrm{mg} / \mathrm{kg}$, which is lower than $0.11-5.85 \mathrm{mg} / \mathrm{kg}$ of Cd observed in soybean leaves, red peppers and rice stalks and leaves at the Daduk Au-Ag-Pb-Zn mine, Korea (Lee et al., 2001), 8.70-12.70 mg/kg observed in Pergularia tomentosa, Calotropis procera, Acacia tortilis, Ochradenus baccatus, Salsola sp., Rhiza strica, Convolvulus sp., Euculeprus sp., Family graminaea, and Prosopis juliflora in Mahad AD'Dahab mine, Saudi Arabia (Al-Farraj and Al-Wabel, 2007) and 2.00-10.00 mg/kg observed in Salsola kali, Andropogon barbinodis, Aster tanacetifolius, Baphia absinthifolia, Asphodelus sp., Solanum elaeagnifolium, and Dyssodia papposa in Zacatecas mine, Mexico (González and González-Chávez, 2006).

Cadmium is a non-essential element for plant metabolism and disturbs symbiosis between microbes and plants, and predisposes plants to fungal invasion (Kabata-Pendias and Pendias, 2001). It (Cd) has no known bio-importance in human biochemistry and physiology and consumption even at very low concentrations can be toxic (Nolan, 2003; Young, 2005) and long term exposure results in renal 
dysfunction, characterized by tubular proteinuria (Duruibe et al., 2007) in humans. Thus, Cd is highly zootoxic and is a common contaminant in the environment (Pulford and Watson, 2003). The concentration of heavy metals especially Cd in Alchornea cordifolia $(2.16 \pm 0.07 \mathrm{mg} / \mathrm{kg})$ and Dialium guinense $(2.02 \pm 0.03 \mathrm{mg} / \mathrm{kg})$ is well above the permissible limit (PL) of $0.2 \mathrm{mg} / \mathrm{kg}$ (Cd) in plants established by Codex Alimentarius Commission FAO/WHO (2007) and this may constitute serious health risk to inhabitants of the mined areas.

Table 5: Comparison of values obtained in this study with permissible limits by FAO/WHO and FEPA

\begin{tabular}{|c|c|c|c|c|c|c|}
\hline $\begin{array}{l}\text { Heavy } \\
\text { Metal }\end{array}$ & $\begin{array}{l}\text { Concentration of } \\
\text { plants in sample } \\
\text { site }(\mathrm{mg} / \mathrm{kg})\end{array}$ & $\begin{array}{l}\text { FAO/WHO } \\
\text { permissible limits } \\
\text { for heavy metals in } \\
\text { plants } \\
(\mathrm{mg} / \mathrm{kg})\end{array}$ & $\begin{array}{l}\text { FEPA } \\
\text { guidelines } \\
\text { for heavy } \\
\text { metals in } \\
\text { plants } \\
(\mathrm{mg} / \mathrm{kg}) \\
\text { (Threshold } \\
\text { values) }\end{array}$ & $\begin{array}{l}\text { Concentration } \\
\text { of soil in } \\
\text { sample } \\
\text { site } \\
(\mathrm{mg} / \mathrm{kg})\end{array}$ & $\begin{array}{l}\text { FAO/WHO } \\
\text { permissible limits for } \\
\text { heavy metals in soil } \\
(\mathrm{mg} / \mathrm{kg})\end{array}$ & $\begin{array}{l}\text { FEPA } \\
\text { guidelines } \\
\text { for } \\
\text { heavy metals in } \\
\text { soils (mg/kg) } \\
\text { (Threshold } \\
\text { values) FEPA } \\
1991 \\
\end{array}$ \\
\hline $\mathrm{Pb}$ & $\begin{array}{l}0.01 \pm 0.00- \\
10.37 \pm 0.52\end{array}$ & $0.3^{\mathrm{b}}$ & NA & $\begin{array}{l}6.11 \pm 0.13- \\
81.60 \pm 0.57\end{array}$ & $50^{\mathrm{b}}$ & 1.6 \\
\hline $\mathrm{Cd}$ & $\begin{array}{l}0.01 \pm 0.00- \\
2.16 \pm 0.07\end{array}$ & $0.2^{\mathrm{a}}$ & NA & $\begin{array}{l}0.01 \pm 0.00- \\
5.02 \pm 0.11\end{array}$ & $3^{b}$ & 0.01 \\
\hline $\mathrm{Ni}$ & $\begin{array}{l}0.01 \pm 0.00- \\
9.02 \pm 0.03\end{array}$ & $1.63^{\mathrm{b}}$ & NA & $\begin{array}{l}4.15 \pm 0.07- \\
79.00 \pm 0.57\end{array}$ & $50^{b}$ & 0.1 \\
\hline $\mathrm{Fe}$ & $\begin{array}{l}0.01 \pm 0.00- \\
231.0 \pm 1.41\end{array}$ & $425^{b}$ & NA & $\begin{array}{l}186.00 \pm 2.83- \\
522.00 \pm 1.98\end{array}$ & NA & 400 \\
\hline As & $\begin{array}{l}0.001 \pm 0.000- \\
0.005 \pm 0.001\end{array}$ & $0.2^{\mathrm{c}}$ & NA & $\begin{array}{l}0.000 \pm 0.000- \\
0.003 \pm 0.000\end{array}$ & $20^{b}$ & NA \\
\hline
\end{tabular}

2001), ${ }^{c}$ FAO/WHO (Codex Alimentarius Commission, 1991), FEPA 1991, NA = Not available

Therapeutic use of $D$. guinense leaves (from the mined sites) on a regular basis for treatment of malaria and similar ailment in the study area will result to $\mathrm{Cd}$ poisoning. The fruit of $D$. guinense is highly consumed in south-eastern Nigeria as a veritable source of vitamin and mineral (Ogbonna et al., 2011). Consequently, households or individuals that have been consuming fruit of D. guinense from the study area may have predisposed themselves to serious health hazard. Heavy metals contamination has great significance due to their tendency to accumulate in human organs over a period of time and its presence beyond the allowed upper and lower limits can cause metabolic disturbance. The environmental impact of heavy metals, as well as their health effects has been the source of major concern (Hussain and Khan, 2010). Iron concentration in plants ranged from $0.01 \pm$ $0.00-231.0 \pm 1.41 \mathrm{mg} / \mathrm{kg}$, which is higher than 153.2-170 mg/kg (Ebong et al., 2008) in a related study while the concentration of As in plants ranged from $0.001 \pm 0.000-0.005 \pm 0.001 \mathrm{mg} / \mathrm{kg}$. The concentrations of As in plants was low and this may be attributed to its concentrations in soil at Akwuke mine (Table 3) and Iva mine (Table 4) sites. This suggests that As in soil was not in the forms (soil solution) that are readily available and absorbable by plants via the roots (Ogbonna et al., 2011). Notwithstanding this, in order to resist the damaging effect of heavy metal in soil, plants adopt different strategies which vary according to the plant genotype (Foy et al., 1978; Di Salvatore et al., 2009). Some of the most effective regulation mechanisms are exclusive, reduce transfer to the shoot of metals adsorbed at the cell walls of the root, chelation and compartmentation of the metals in the vacuole through the production of organic acids and formation of metal binding polypeptides known as phytochelatins (Hall, 2002).

Generally, the highest concentration of heavy metals accumulated in wet season were significantly ( $P$ $<0.05)$ higher than their corresponding values in dry season. Indeed, while several studies showed seasonal changes in heavy metal content in plants, others found no seasonal changes in metals (Gleason et al., 1979; Larsen and Schierup, 1981; Cacador et al., 2000; Weis et al., 2003). Consequently, it is very difficult to generalize about seasonal changes in heavy metal levels, since they appear to vary greatly with the heavy metal and species of plants studied (Weis and Weis, 2004).

\subsection{Conclusion}

This study indicated that the levels of heavy metals accumulated in the plant species were higher in wet season than in dry season. Plant samples from the coal mine sites recorded significant levels of heavy metals but the concentration of $\mathrm{Ni}, \mathrm{Pb}$ and $\mathrm{Cd}$ accumulated in A. cordifolia and A. gayanus was 
higher than the permissible limits established by the Codex Alimentarius Commission FAO/WHO safe limit. Further studies should be carried out to ascertain other heavy metals present at the mined sites.

\section{Acknowledgement}

We thank Mrs. I.L. Princewill-Ogbonna for the encouragement and support she provided during the field visit and sample collection at the Akwuke and Iva coal mine sites.

\section{References}

Ademoroti, C.M.A., (1996). Heavy metals in air. Foludex Press, Ibadan, Nigeria.

Angelova, V., et al. (2004). Bioaccumulation and distribution of heavy metals in fibre crops (flax, cotton and hemp). Industrial Crops and Products, 19(3), pp. 197-205.

Al-Farraj A.S. and Al-Wabel, M.I., (2007). Heavy metals accumulation of some plants species grown on mining area at Mahad AD'Dahab, Saudi Arabia. Journal of Applied Sciences, 7(8), pp. 1170-1175.

Alloway, B.J., (1995). Heavy metals in soils. Blackie and Son Limited, Glasgow, United Kingdom.

Alloway, B.J. and Ayres, D.C., (1997). Chemical principles of environmental pollution. Black Academic and Professional Publication, London. United Kingdom.

Awofolu, O.R., (2005). A survey of trace metals in vegetation, soil and lower animals along some selected major and minor roads in metropolitan city of Lagos. Environmental Monitoring and Assessment, 105, pp. 431-447.

Berman, E. (1980). Toxic metals and their analysis. Heyden and Sons, London, United Kingdom.

Boonyapookana, B., et al. (2005). Phytoaccumulation of lead by sunflower (Helianthus annus), Tobacco (Nicotiana tabacum), and Vetiver (Vetiveria zizanioides). Journal of Environmental Science and Health Part A, 40(1), pp. 117-137.

Borg, H. and Johansson, K., (1989). Metal fluxes to Swedish forest lakes. Water Air Soil Pollution, 47, pp. 427-440.

Cacador, I., et al. (2000). Seasonal variation of $\mathrm{Zn}, \mathrm{Pb}, \mathrm{Cu}$, and $\mathrm{Cd}$ concentrations in the rootsediment system of Spartina maritima and Halimione portulacoides from Tagus estuary salt marshes. Marine Environmental Research, 49(3), pp. 279-290.

Chang, P., et al. (2005). Concentrations of arsenic and heavy metals in vegetation at two abandoned mine tailings in South Korea. Environmental Geochemistry and Health, 27(2), pp. 109-119.

Codex Alimentarius Commission FAO/WHO (2007). Joint FAO/WHO Food Standard Programme 13th Session. Report of the Thirty Eight Session of the Codex Committee on Food Hygiene, Houston, United States of America,ALINORM 07/30/13.

Deram, A., et al. (2006). Seasonal variations of cadmium and zinc in Arrhenatherum elatius, a perennial grass species from highly contaminated soil. Environmental Pollution, 140(1), pp. 62-70.

Di-Salvatore, M., et al. (2009). Assessment of heavy metals transfer from a moderately polluted soil into the edible parts of vegetables. Journal of Food, Agriculture and Environment, 7(2), pp. 683-688.

Dudka, S. and Adriano, C., (1997). Environmental impact of metal mining and processing. Journal of Environmental Quality, 26(3), pp. 590-602. 
Duruibe, J.O., et al. (2007). Heavy metal pollution and human biotoxic effects. International Journal of Physical Sciences, 2(5), pp. 112-118.

Ebong, G.A., et al. (2008). Heavy metal contents of municipal and rural dumpsite soils and rate of accumulation by Carica papaya and Talinum triangulare in Uyo. E-Journal of Chemistry, 5(2), pp. 281-290.

Ekere, N.R. and Ukoha, O.P., (2013). Heavy metals in street soil dusts of industrial market in Enugu, south east, Nigeria. International Journal of Physical Sciences, 8(4), pp. 175-178.

Ezeigbo, H.I. and Ezeanyim, B.N., (1993). Environmental pollution from coal mining activities in the Enugu area, Anambra State, Nigeria. Mine Water and the Environment, 12(1), pp. 53-62.

Foy, C.D., et al. (1978). The physiology of metal toxicity in plants. Annual Review of Plant Physiology, 29, pp. 511-566.

Gleason, M.L., et al. (1979). Seasonal and environmental variation in $\mathrm{Mn}, \mathrm{Fe}, \mathrm{Cu}$ and $\mathrm{Zn}$ content of Spartina alterniflora. Aquatic Botany, 7, pp. 385-392.

Gonzalez, R.C. and Gonzalez-Chavez, M.C.A., (2006). Metal accumulation in wild plants surrounding mining waste. Environmental Pollution, 144(1), pp. 84-92.

Hall, J.L., (2002). Cellular mechanisms for heavy metal detoxification and tolerance. Journal of Experimental Botany, 53(366), pp. 1-11.

Hunter, J.G. and Vergnano, O., (1952). Nickel toxicity in plants. Applied Biology, 39, pp. 279-284.

Hussain, I. and Khan, L., (2010). Comparative study on heavy metal contents in Taraxacum officinale. International Journal of Pharmacognosy and Phytochemical Research, 2(1), pp. 15-18.

Hutton, M., (1986). Human health concerns of lead, mercury, cadmium and arsenic. In: Hutchinson, T.C. and Meema, K.M. (eds), lead, mercury, cadmium and arsenic in the environment. John Wiley and Sons, Brisbane.

Jung, M.C., (2008). Heavy metal concentrations in soils and factors affecting metal uptake by plants in the vicinity of a Korean Cu-W mine. Sensors, 8(4), pp. 2413-2423.

Kabata-Pendias, A. \& Pendias, H., (2001). Trace elements in soils and plants. CRC Press, Boca Raton.

Kabata-Pendias, A., (2000). Trace Elements in Soils and Plants. CRC Press, Boca Raton.

Khan, D.A., et al. (2008). Lead-induced oxidative stress adversely affects health of the occupational workers. Toxicology and Industrial Health, 24, pp. 611-618.

Larsen, V.J. and Schierup, H.H., (1981). Macrophyte cycling of zinc, copper, lead, and cadmium in the littoral zone of a polluted and a non-polluted lake II. Seasonal changes in heavy metals content of above-ground biomass and decomposing leaves of Phragmites australis (Cav.). Trinidad. Aquatic Botany, 11, pp. 211-230.

Lee, C.G., et al. (2001). Heavy metal contamination in the vicinity of the Daduk Au-Ag-Pb-Zn mine in Korea. Applied Geochemistry, 16(11-12), pp. 1377-1386.

Li, J., et al. (2006b). Risk assessment for safety of soils and vegetables around a lead/zinc mine. Environmental Geochemistry and Health, 28(1-2), pp. 37-44. 
Li, Y., et al. (2006a). Risk assessment of heavy metals in soils and vegetables around non-ferrous metals mining and smelting sites, Baiyin, China. Journal of Environmental Science, 18(6), pp. 11241134.

Mac Farland, H.N., (1979). Pulmonary effects of cadmium: In Mennea, J.E. (ed), cadmium toxicity, Dekker.

Manzur, R.T., (1981). Minerals and man. Austolasian Inst. Mag. Wet Portville, Australia.

McGrath, S.P., et al. (2001). Plant and rhizosphere process involved in phytoremediation of metalcontaminated soils. Plant Soil, 232(1-2), pp. 207-214.

Mtui, G.Y.S., et al. (2006). Vegetation structure and heavy metal uptake by plants in the miningimpacted and non-mining impacted sites of southern lake Victoria Wetlands. Tanzania Journal of Science, 32(2), pp. 39-49.

National Population Commission of Nigeria (NPC) (2006). Population and Housing Census Result. NPC, Lagos.

Nolan, K., (2003). Copper toxicity syndrome. Journal of Orthomolecular Psychiatry, 12(4), pp. 270282.

Noret, N., et al. (2007). Do metal-rich plants deter herbivores? A field test of the defence hypothesis. Oecologia, 152(1), pp. 92-100.

Ogbonna, P.C., et al. (2011). Heavy metal concentration in soil and woody plants in a quarry. Toxicological and Environmental Chemistry, 93(5), pp. 895-903.

Ogbonna, P.C., et al. (2012). Heavy metal concentration in soil and plants at a coal mine. Terrestrial and Aquatic Environmental Toxicology, 6(2), pp. 127-131.

Ogbonna, P.C., et al. (2018a). Soil chemical characteristics in wet and dry season at Iva long wall underground mined site, Nigeria. Nigerian Journal of Environmental Sciences and Technology, 2(1), pp. 96-107.

Ogbonna, P.C., et al. (2018b). Heavy metals in soil and accumulation in medicinal plants at an industrial area in Enyimba city, Abia State, Nigeria. Nigerian Journal of Environmental Sciences and Technology, 2(1), pp. 89-95.

Ogbonna, P.C., et al. (2018c). Seasonal variation of soil chemical characteristics at Akwuke long wall underground mined site, Nigeria. Journal of Applied Sciences and Environmental Management, 22(8), pp. $1303-1310$.

Ogbonna, P.C. and Okeke, V., (2011). Heavy metal level of soil and gmelina plantation in Umuahia, Nigeria. Terrestrial and Aquatic Environmental Toxicology, 5(1), pp. 31-34.

Ogwuegbu, M.O.C. and Muhanga, W., (2005). Investigation of lead concentration in the blood of people in the copperbelt province of Zambia. Journal of Environment, 1, pp. 66-75.

Pam, A.A., et al. (2014). Heavy metals contents in soils and some crops irrigated along the Bindare Stream Zaria- Kaduna State, Nigeria. American Chemical Science Journal, 4(6), pp. 855-864.

Perez-Sirvent, C., Navarro, M.C., Martinez-Sanchez, M.J., Vidal J., Tovar, P.J., et al. (2008). Abandoned mine sites as a source of contamination by heavy metals: A case study in a semi-arid zone. Journal of Geochemical Exploration, 96(2), pp. 183-193. 
Pokethitiyook, P., et al. (2008). Spatial and seasonal variations in lead content of plants colonizing the Bo Ngam lead mine, Thailand. Science Asia, 34, pp. 169-178.

Pruvot, C., et al. (2006). Heavy metals in soil, crops and grass as a source of human exposure in the former mining areas. Journal of Soils and Sediments, 6(4), pp. 215-220.

Pulford, I.D. and Watson, C., (2003). Phytoremediation of heavy metal-contaminated land by treesa review. Environment International, 29(4), pp. 529-540.

Raskin, I., et al. (1997). Phytoremediation of metals: Using plants to remove pollutants from the environment. Current Opinion in Biotechnology, 8(2), pp. 221-226.

Reeves R.D. \& Baker A.J.M., (2000). Metal accumulating plants. In: Raskin, I., Ensley, B.O. (eds), phytoremediation of toxic metals: Using plants to clean up the environment. John Wiley and Son Inc., New York, USA.

Singh, B., et al. (2010). Micronutrient deficiency: A global challenge and physiological approach to improve grain productivity under low zinc availability. In: Plant Nutrition and Abiotic Stress Tolerance II (Anjum N A, ed). Plant Stress, 4, pp.76-93.

Steel, R.G.D. and Torrie, J.H., (1980). Principles and procedures of statistics: A biometric approach, McGraw-Hill, New York.

Stoltz E. and Greger, M., (2002). Accumulation properties of As, $\mathrm{Cd}, \mathrm{Cu}, \mathrm{Pb}$, and $\mathrm{Zn}$ by four wetland plant species growing on submerged mine tailings. Environmental and Experimental Botany, 47(3), pp. 271-280.

Udedi, S.S., (2003). From guinea worm scourge to metal toxicity in Ebonyi State, Chemistry in Nigeria as the New Millenium unfolds.

Weis, J.S. and Wees, P., (2004). Metal uptake, transport, and release by wetland plants: Implications for phytoremediation and restoration. Environment International, 30(5), pp. 685-700.

Weis, J.S., et al. (2003). Patterns of metal accumulation in leaves of the tidal marsh plants Spartina alterniflora Loisel and Phragmites australis (Cav.). Trinex Steud, 23, pp. 459-465.

Yang Q.W., et al. (2006). Cadmium in soil-rice system and health risk associated with the use of untreated mining wastewater for irrigation in Lechang, China. Agricultural Water Management, 84, pp. 147-152.

Young, R.A. (2005). Toxicity profiles: Toxicity summary for cadmium. Risk Assessment Information System, RAIS, University of Tennesse. (rais.ornl.gov/tox/profiles/cadmium.shtml).

Zheng, N., et al. (2007). Health risk of $\mathrm{Hg}, \mathrm{Pb}, \mathrm{Cd}, \mathrm{Zn}$, and $\mathrm{Cu}$ to the inhabitants around Huludao Zinc Plant in China via consumption of vegetables. Science of the Total Environment, 383, pp. 81-89.. 